\title{
Link up Industry 4.0 with the Enterprise Collaboration System to Help Small and Medium Enterprises
}

\author{
Hua-Ko Chiang, Yukari Nagai $\mathbb{D}^{\text {, }}$, and Yung-Yu Lin \\ School of Knowledge Science, Japan Advanced Institute of Science and Technology, Nomi City, Japan \\ Correspondence should be addressed to Yukari Nagai; yukarinagai.jp@gmail.com
}

Received 23 June 2020; Accepted 31 July 2020; Published 10 September 2020

Guest Editor: Ching-Hsin Wang

Copyright (c) 2020 Hua-Ko Chiang et al. This is an open access article distributed under the Creative Commons Attribution License, which permits unrestricted use, distribution, and reproduction in any medium, provided the original work is properly cited.

\begin{abstract}
Along with Industry 4.0 and the Internet of Things (IoT), the need for e-management has been extensive. For helping small and medium enterprises (SMEs) to take the first step to link up Industry 4.0, the implementation of the enterprise collaboration system (ECS) in SMEs has become inevitable. We use SuccMail to examine the effect of external supports and internal supports for ECS implementation of performance. To conduct this aim, we adopted the questionnaire from ERP and revised it for ECS. We collected the data from three SMEs from Taiwan and used the SPSS to run the correlation and multiple regressions. We find that all the supports are related to the performance; however, considering all the supports together, only some supports have significant and positive effects. We conclude that the supports can help SMEs in implementing ECS to increase the performance. Furthermore, the internal supports are more significantly and positively affecting the performance of implementing ECS in SME.
\end{abstract}

\section{Introduction}

The Enterprise Collaboration System (ECS) is a kind of application software that supports collaboration and management in enterprises. Along with Industry 4.0 and the development of the Internet of Things (IoT), ECS has been of growing importance for SME to take the first step of linking up Industry 4.0. To improve the performance of SMEs, many features are provided from ECS for cost reduction, improving productivity, avoiding potential failures, and defect diagnosis. In developed and developing countries, SMEs account for the major source of employment, technological advancements, and competitive advantages [1]. Information systems (IS) have become a vital component of an organization's competitive practices [2]. The competitive pressure and necessity of SMEs getting to enter the global market are increasing, and SMEs are increasingly adopting Information Technology (IT) to take advantage of its substantial benefits. Social Media and Enterprise Collaboration Systems are both based on social software and are thus similar in functionality [3], and, according to Suh and Bock [4], the use of ESM (Enterprise Social Media) leads to better task performance. Most of the prior research studies have focused more on IT adoption in large organizations [5-7].

On the other hand, due to the limited resources controlled by SMEs, the process of IT adoption by SMEs is considerably different [1]. Most of the SMEs are using the features afforded by free applications (e.g., LINE, Outlook, and Dropbox). Nowadays, there is a lot of software made for SMEs, and most of that software has the following features: the messages storage, file sharing, data searching, crossplatform, etc. The system software with such features is classified as ECS. Most of the ECSs only provide basic features for free. Table 1 compares the features of some ECS, including SuccMail, Slack, Teams, JANDI, WorkDo, Yammer, LINE, Outlook, and Dropbox. However, there is a lack of research on examining those ECS, not to mention the overall comparison.

In this paper, we focused on studies on an ECS software, "SuccMail." The reason for choosing SuccMail instead of widely used Slack or Teams is because SuccMail has the same basic features that Slack and Teams have. Also, the SuccMail founder is an entrepreneur and the core concept of SuccMail is starting as managers and simplifying the complex. 
TABLE 1: The comparison of ECSs and freeware apps.

\begin{tabular}{|c|c|c|c|c|c|c|c|c|c|c|c|c|}
\hline & \multicolumn{2}{|c|}{ Message } & \multirow{2}{*}{$\begin{array}{c}\text { File sharing } \\
\text { File }\end{array}$} & \multicolumn{3}{|c|}{ Search } & \multicolumn{3}{|c|}{ Administration } & \multicolumn{3}{|c|}{ Cross-platform } \\
\hline & One-on-one & Group & & Message & File & Filter & Invite & Remove & $\mathrm{PC}$ & $\mathrm{Mac}$ & Android & iOS \\
\hline SuccMail & $\mathrm{O}$ & 0 & 0 & $\mathrm{O}$ & 0 & $\mathrm{O}$ & O & 0 & 0 & $\mathrm{O}$ & O & O \\
\hline Slack & O & O & O & O & O & O & ○ & O & O & O & O & O \\
\hline Teams & O & O & O & O & O & O & O & O & 0 & O & O & O \\
\hline JANDI & O & O & O & O & O & O & O & O & 0 & O & O & ○ \\
\hline WorkDo & O & O & O & O & O & O & O & O & 0 & O & O & O \\
\hline Yammer & O & O & O & O & O & O & O & O & 0 & O & O & ○ \\
\hline LINE & O & O & O & O & $x$ & $x$ & O & $x$ & 0 & O & O & O \\
\hline Outlook & O & O & $x$ & O & $x$ & O & $x$ & $x$ & O & O & O & O \\
\hline Dropbox & $x$ & $x$ & 0 & $x$ & 0 & $x$ & 0 & 0 & 0 & 0 & 0 & 0 \\
\hline
\end{tabular}

Therefore, we investigate three SMEs from Taiwan using SuccMail: one multinational trading company, one trading company, and one education center. The questionnaire design for these three SMEs includes three parts: external support, internal support, and performances. This paper mainly studies an ECS, "SuccMail." Therefore, in this paper, it is hard to distinguish ECS and SuccMail. We suggest that it is reasonable to consider SuccMail as ECS or ECS as SuccMail in this paper. We suppose that the number of employees in the SME that uses ECS is from 10 to 50. Here, the employees are referred to as those who work in front of the computer or who need to use the computer to work every day.

Therefore, the objective of this research is to elucidate the impact and the performance after enterprise implements "SuccMail." To attain this objective, our research will answer 1 Major Research Question (MRQ) and 2 Subsidiary Research Questions (SRQs). The MRQ is, how does the support of SuccMail can affect the performance of SME? SRQ 1 is, how does the internal support from SME affect the performance of SME? SRQ 2 is, how does the external support (from SuccMail of the ECS) affect the performance of SME?

As mentioned above, the paper is organized into seven sections. Following the introduction, we will deal with the literature on ECS in SMEs and explain why the enterprises that have ERP, e-mail, and Instant Messaging still need ECS. Also, we will introduce the design features of SuccMail. Then we will present our research model and hypotheses. In the research, methodology, the research strategy, data collection, data source, and measurement of items will be discussed. Finally, following the results, we will give the discussion and conclusion.

\section{Literature Review}

The concept of ECS is arising from e-commerce development and application demand [8]. ECS is the software, which has essential knowledge management features. Employees can easily understand today's work content and the required knowledge through ECS; any employee can get in touch with other members in real time, find experts who can help, or quickly connect to the related portal [9].

2.1. Enterprise Collaboration System. ECS is a sociotechnical system that includes hardware, software, and internal and external networks, as well as people, processes, and organizational aspects [10]. The ECS allows people to work together on the Internet in real time. We consider that ECS should have three basic concepts: communication, coordination, and collaboration [11-13]. Some of the others argued that the project management systems are ECSs as well. However, it is hard to give a narrow definition of the ECS. Broadly speaking, ECS is the integration with Enterprise Social Software (ESS) components (e.g., search, links, post, and tags) [14], communication system, and knowledge management system to support collaboration and management in the organization.

2.2. The Reasons Why Enterprises Having ERP Still Need ECS. Both of the systems ERP and ECS require user skills. Generally speaking, the skills required for ERP are stick and routine. Meanwhile the skills required of ECS are more flexible, requiring the user to understand the suitability of a tool for a current task in hand and to make appropriate selections. ERP systems are based on a process-oriented view [15] to support clearly defined and repeatable business features with built-in business rules. ERP systems are critical to enterprises because they support the core production process. For the users, the first important difference concerns of ERP are their application area and the structure of their content. ECS, on the other hand, is designed to support joint work among people in the workplace.

According to Chou and Rouhani [16, 17], from their experience, a rate between $65 \%$ and $85 \%$ failure of ERP system and less than $70 \%$ of the ERP system capabilities were used in the organizations. Futhermore, the enterprise can only get less than $35 \%$ of promised benefits from ERP system [18]. The advanced version of SuccMail is in progress of linking ERP with ECS. The advanced version of SuccMail is in progress, we are evaluating the performance of linking ERP to SuccMail.

In large enterprises, ECS for SMEs is like the Enterprise Information Portal (EIP) system of large enterprises. Even though the SMEs successfully implement ERP and operate smoothly, unlike large enterprises, they do not have enough resources to develop their own ERP and EIP system. There is a fundamental difference between the software of ECS and ERP. ECS supports communication, coordination, and collaboration in the enterprise. The user of ECS is often a volunteer who must be convinced of the benefits of using these tools. This is why "user acceptance" has traditionally 
played a much bigger role in research on collaboration systems [14]. However, ERP data comprise highly structured master data and transactional data reflecting the enterprise's resources and business activities, while most parts of ECS contain unstructured contents such as documents, emails, messages, or enterprise instructions. They also differ in purpose and use. ERP gives little room for creativity, imposes structure, and implements the order of events onto the user. The use of ERP systems is normally mandatory. ECSs, on the other hand, are tools for ad-hoc use which offer choice and thus entail uncertainty [15].

\subsection{The Reasons Why Enterprises Having E-Mail and Instant} Messaging Still Need ECS. Nowadays enterprises have a lot of different choices to communicate. Most companies use a wide variety of mediums to communicate with their employees and customers, such as e-mail, Skype, LINE, WhatsApp, and WeChat. The most popular communication medium is e-mail, while e-mail has become an unproductive tool. The research introduces three facets of information overload in e-mail communication: a large amount of incoming information, inefficient workflow, and deficient communication quality [19]. The typical corporate user spends 2 hours and 14 minutes every day reading and responding to e-mails [20].

"Email has its place but not as a catch-all communications tool," says global managing director for R\&D at consultancy Accenture. For example, e-mail was never designed for real-time communications as anyone who has tried to urgently get hold of a colleague by e-mail will know [21]. The extensive reliance on e-mail has led to numerous problems, studied extensively by CSCW researchers. Users face difficulties in dealing with the high number of messages-e-mail overload, and e-mail is often only indirectly useful for the actual task at hand.

These days, there are a lot of Instant Messaging (IM) apps on the market. Most of them are free to use, even for the enterprise. Hence, a lot of SMEs use IM to communicate not only in the enterprise but also with the customer. There is a lot of researches about IM for enterprises, and most of them agreed that using IM has benefits for SMEs. As previous researches, we agree it has benefits for SMEs. However, we believe that managing the SMEs only with IM is not enough. SMEs need other software to work with IM to achieve better performance in management. Therefore, to improve the communication and management quality of the enterprise, an appropriate tool is very important and necessary.

2.4. The Design Features of SuccMail. SuccMail is designed for SMEs, and all the following features are designed to manage SMEs.

2.4.1. Accessibility of Historical Messages by Any Group Member. When assigned as a group member, you have the accessibility to read any historical messages in this group. The purpose of these features is to help all employees to understand the relevant pieces of information from past events.
2.4.2. Customizable Labels Based on Events and Objects for Search Feature. Every enterprise has its unique events and objects labels. To unify the terminology in the enterprises, the labels are very important keywords. Any historical messages can be easily searched by the event or object label. Figure 1 shows a customizable search; there are some selections of filter to choose. The filter selection includes keyword, date range, group, and the document. Figure 2 shows the results of the search from Figure 1.

2.4.3. Display of Messages Status (Who Read/Unread the Messages and When). Most of the time we send out the messages or e-mail expecting to receive the response as soon as possible. However, we do not know when does the receiving person read it or even receive it. Therefore, with the display of messages state, the user can clearly understand whether the messages have been read by assigned coworkers. Figure 3 shows that Member B answers the message sent by Member $A$ on $1 / 12$ at $14: 32$ on $1 / 12$ at 17:20 and Member A read this message on $1 / 12$ at 17:33, while Member $C$ has not read the message yet.

2.4.4. Tracking Ongoing or Completed Events. To prevent making all the events stack together, SuccMail classifies the events that had been done to "Completed Area" to separate from the ongoing events. All members can apply to file the event to "Completed Area," while only the authorized platform manager can archive the completed events. Furthermore, any filed events in the "Completed Area" can be returned to the "Ongoing Area" to be discussed again by any group members. Figure 4 shows the status of the ongoing events, and Figure 5 shows the status of the completed events.

2.4.5. Links to Any Events by a Unique URL. Every event has a unique Uniform Resource Locator (URL). The user can easily link any event with a unique URL no matter it is an ongoing or completed event. Figure 6 shows the producing area of the resume URL, which can be linked to any event.

\section{Theory and Hypotheses}

3.1. Research Model. According to Greeven and Williams [22], the SMEs that implement ECS will face a lot of challenges. The top management support influences successful IS implementation [23]. Successful implementation of any system, organizational support is indispensable [24]. Therefore, we considered that the supports and resources can help SMEs to face the challenges while implementing ECS as our hypothesis. We classified the supports and resources into internal support and external support. Figure 7 is our research model. The support that employees can gain from inside of SMEs is the internal support and the support that employees can gain from the ECS side is external support. The benefit of using ECS can be expected [25], while SMEs often lack a clear vision of benefit [3]. Since the benefits are not always reflected in finances, in our hypothesis, we consider the benefits of using ECS as the 


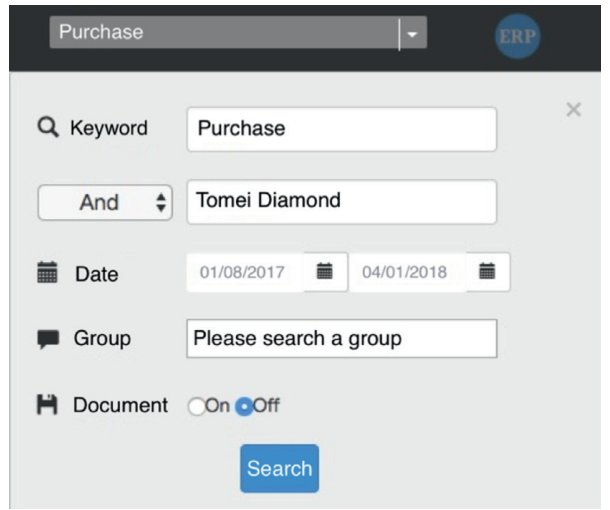

FIgURE 1: The customizable search.

\section{Search Result}

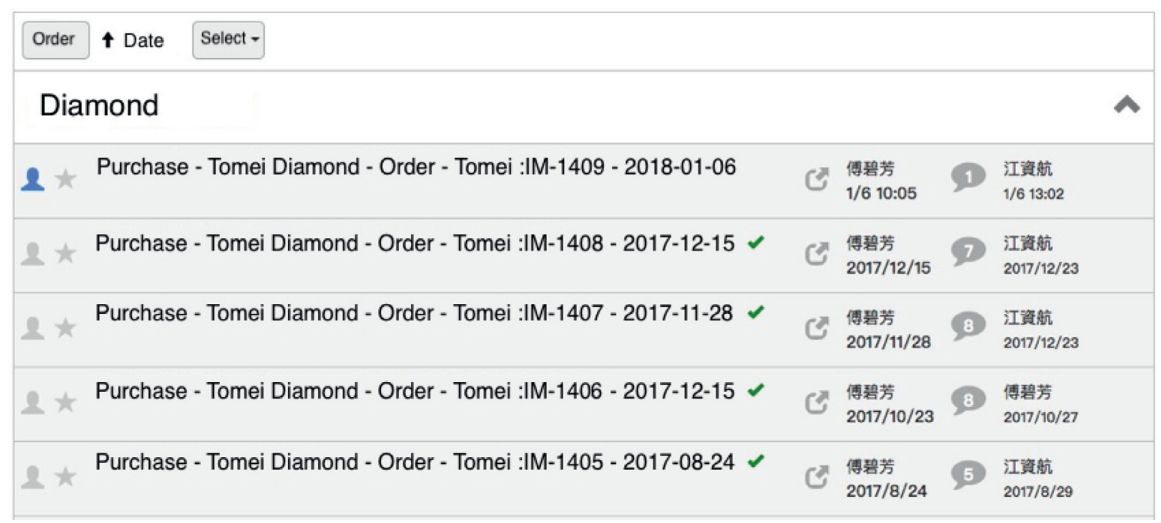

FIgURE 2: The search result of Figure 1.

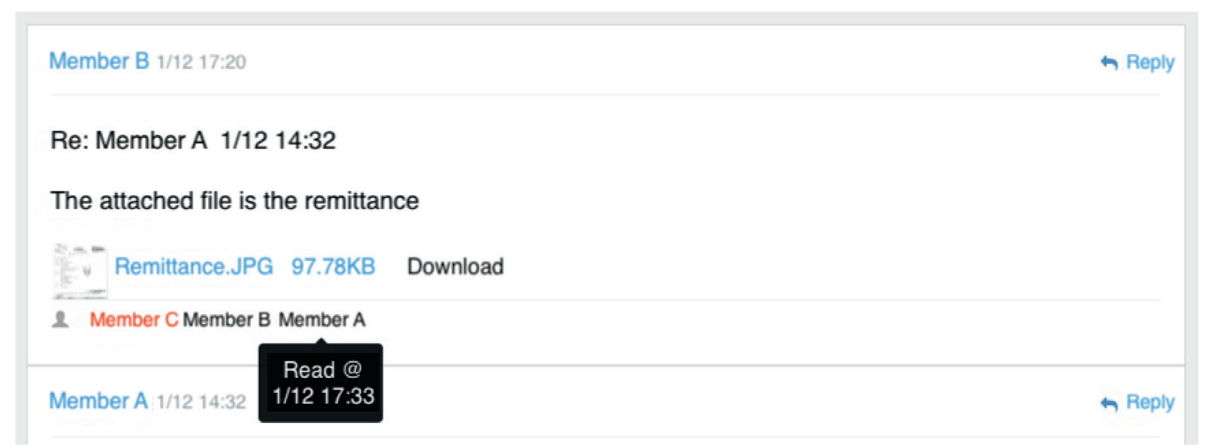

Figure 3: The messages state.

performance of using ECS. We believe that the performance of using ECS includes the following:

(1) Using SuccMail is easier to perform tasks (P1)

(2) SuccMail is helpful to improve communication (P2)

(3) SuccMail is helpful to reduce the cost of human resources $(\mathrm{P} 3)$

(4) SuccMail is helpful to reduce customer complaints (P4)
(5) Using SuccMail can enhance working efficiency (P5)

(6) Using SuccMail can increase production efficiency (P6)

3.2. Hypotheses. ECS is flexible and malleable software and the work of ECS is dependent on employees' skills and experience $[26,27]$. If the employees do not have enough relevant skills and experience or if they are facing any challenge, the external support can be able to help them get 


\section{General Information}

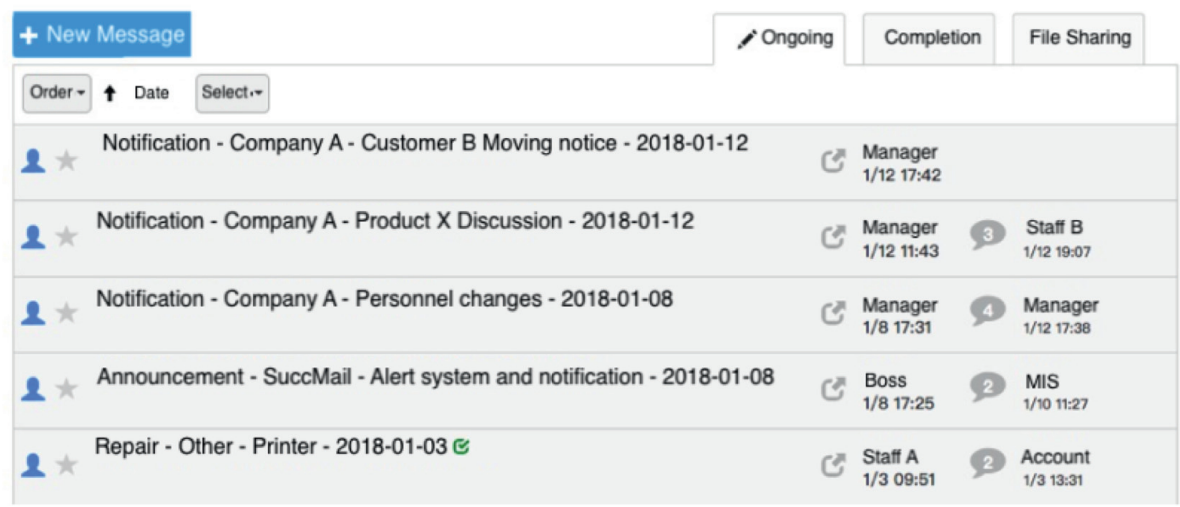

FIgURE 4: The ongoing events.

\section{General Information}

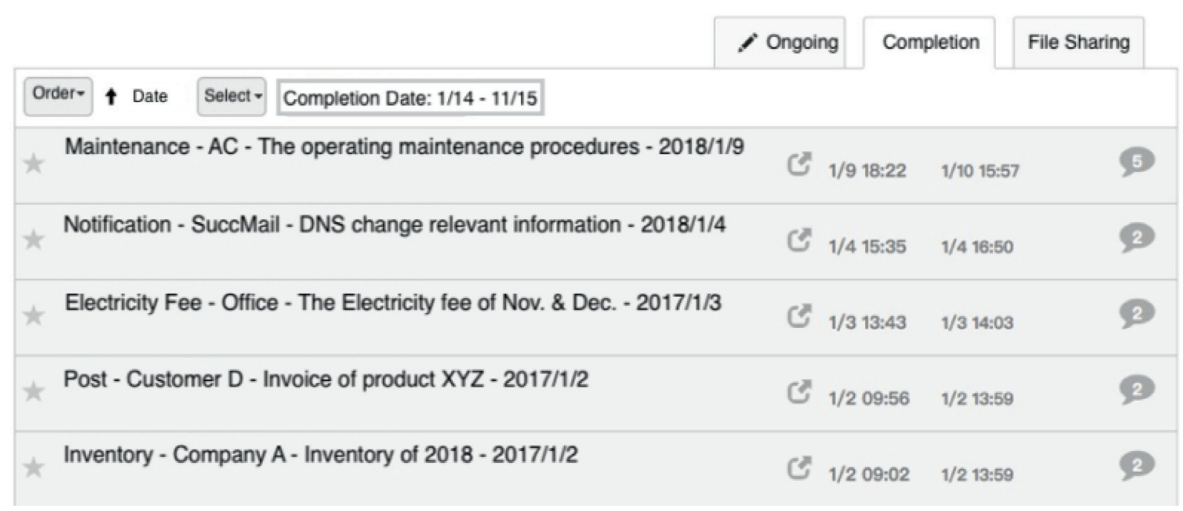

Figure 5: The completed events.

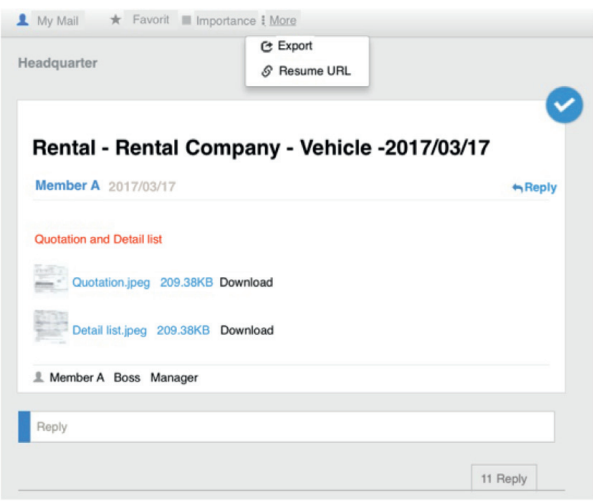

Figure 6: The URL link.

through the challenge in the implementation of ECS. The supports that can be provided from the ECS include software manuals, online consultation, and consultant. Therefore, we have the following hypotheses:

(1) Hypothesis 1 (E1). The SuccMail manual is helpful to use, which has a positive effect on the performance of implementing ECS.
(2) Hypothesis 2 (E2). Online consultation is helpful to solve users' problems of SuccMail, which has a positive effect on the performance of implementing ECS.

(3) Hypothesis 3 (E3). An external SuccMail expert consultant helps use SuccMail, which has a positive effect on the performance of implementing ECS.

The support of SME managers, assistance to employees, and a relatively high initial utilization rate can increase the success rate of ECS implementation [22]. Therefore, we have the following hypotheses:

(4) Hypothesis 4 (I1). The enterprise fully depends on using SuccMail for management and communication, which has a positive effect on the performance of implementing ECS.

(5) Hypothesis 5 (I2). The enterprise offers sufficient resources for using SuccMail, which has a positive effect on the performance of implementing ECS.

(6) Hypothesis 6 (I3). The enterprise hires a full-time consultant for implementing SuccMail, which has a positive effect on the performance of implementing ECS. 


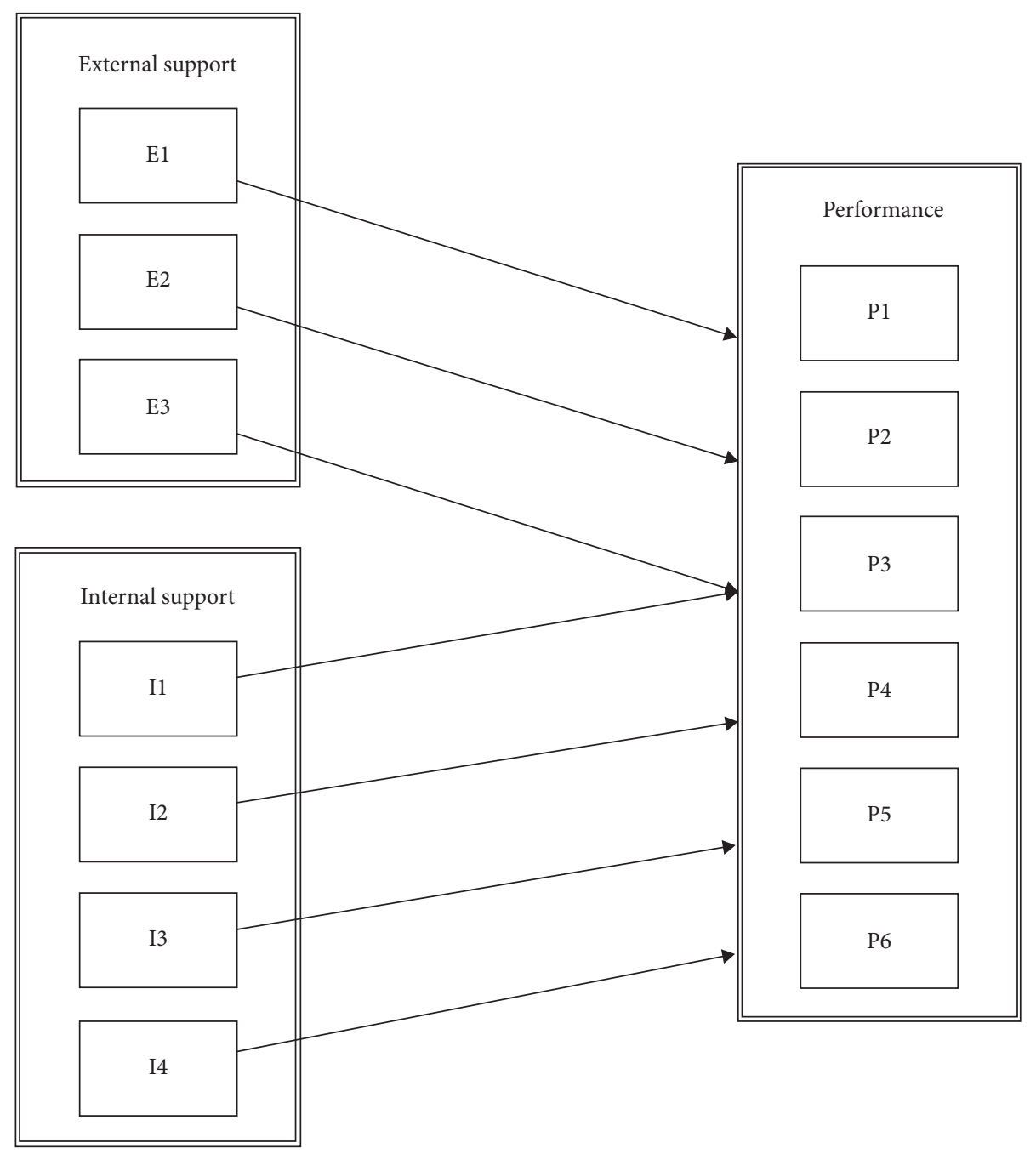

Figure 7: The research model.

(7) Hypothesis 7 (I4).The enterprise conflicts have been reduced by using SuccMail, which has a positive effect on the performance of implementing ECS.

\section{Research Methodology}

4.1. Research Strategy. To improve the accuracy, validity, and stability of the survey results, we use multiple cases as a research goal [28]. The evidence from multiple cases is often considered more compelling, and the overall study is therefore regarded as being more robust [29]. For considering the different types of SME, this study selected three companies: a multinational trading company, a trading company, and an education center. From the perspective of methodology, we try to determine the overarching goal by exploratory factor analysis (EFA) to identify the underlying relationships between measured variables (effectiveness) and also identify the internal support and external support as two sets of latent constructs. Furthermore, this methodology also gives an exploration to discuss the interrelation among support and performance.
The structured questionnaire was used in our research for data collection. Since there are few studies on ECS, we adopted some ERP literature reports as references, which have the same characters as ECS [22], we adopt it from the literature reports of ERP, which have the same characters as ECS. Indeed, we are aware that there is a lack of samples. In order to ensure the validity and reliability of each sample, we chose 3 different-sized SMEs. The numbers of SuccMail users are 10,18, and 34; also they have been using SuccMail for more than 2 years. We keep tracking their usage of SuccMail. Meanwhile, besides this survey, we conducted one-on-one interviews with all the respondents in the past year at least 3 times. To make the questionnaire follow the ECS, we modified it based on the uniqueness and specialty of the ECS. The draft of the questionnaire was pretested by five experts (e.g., ECS founder, ECS advisor...) to validate it, and we sought all experts' opinions to improve it until all of them accepted it.

4.2. Data Collection. The data were collected from 62 users of three SMEs that have been implementing "SuccMail" 
(ECS) for more than one year before our study. The enterprises were identified from the ECS vendor SuccMail.

4.3. Data Source. All of the survey questionnaires were personally sent to 62 SuccMail users from 3 enterprises straightly. For surveys of the questionnaire, we had reached out with the owner or the person who is in charge of the enterprises and made an appointment with each enterprise. The overall recovery ratio of the questionnaire is $62(100 \%)$. None of the questionnaires were invalid.

Demographics of the respondents and companies showed that $34(54.8 \%)$ respondents were from $\mathrm{A}$, the multinational trading company, 18 (29.1\%) were from B, the education center, and $10(16.1 \%)$ were from $\mathrm{C}$, the trading company. There were 10 (16\%) employers, $19(31 \%)$ general managers or deputy general managers, 24 (36\%) staff, and 9 (14\%) teachers. The reason why there were only three enterprises having 10 employers is because they were cofinancing and operating.

$24(39 \%)$ of the respondents were male and $38(61 \%)$ were female. $40(64 \%)$ respondents were in their twenties and thirties, $12(20 \%)$ were in their forties, and $10(16 \%)$ were over fifties. $2(3 \%)$ had work experience less than 5 years, 12 (20\%) had 5-9 years, $9(14 \%)$ had $10-14$ years, $22(36 \%)$ had 15-19 years, and 17 (27\%) had more than 20 years' work experience. 12 (20\%) were high school graduates, 33 (53\%) had a bachelor's degree, 15 (24\%) had a master's degree, and 2 (3\%) had a Ph.D. degree.

4.4. Measurement of Items. The full questionnaire is given in Appendix A, which contains two parts. The first section has 5 fundamental profiles that are the main data source of the descriptive statistics and asking who uses the SuccMail. The second part has 3 dimensions. One is the evaluation of external support for SuccMail, which has three questions, $\mathrm{E} 1, \mathrm{E} 2$, and E3, another is the internal support from the enterprise, which has four questions, I1, I2, I3, and I4, and the last one relates to the performance of implementing SuccMail, which has six questions from P1 to P6.

4.5. Validity and Reliability Tests. The validity and reliability tests were conducted on all the questions from three dimensions as a reference for the next step. We adopted analysis to run the Varimax rotation to test the convergent validity. Table 2 shows that the KMO value is 0.822 and Bartlett's test of Sphericity is significant [30], and Table 3 shows that all the loading values of dimensions for the relevant latent factors were greater than 0.4 , verifying the convergent validity of all the questions [31].

Table 4 shows that the results of Cronbach's alpha value are $0.859,0.842$, and 0.776 . Through the Cronbach alpha value from the measurement items of the questionnaire we test whether the scales are stable and reliable. Reliability tests were run to ensure that the results of the measurement items are stable and consistent. This research adopts the Cronbach alpha value $>0.700$, which is reliable [32].
TABLE 2: KMO and Bartlett's test results.

\begin{tabular}{lcc}
\hline Kaiser-Meyer-Olkin measure of sampling adequacy & 0.822 \\
\hline & Approx. chi-square & 447.469 \\
Bartlett's test of Sphericity & Df & 78 \\
& Sig. & 0.000 \\
\hline
\end{tabular}

TABLE 3: The exploratory factor analysis rotated-component matrix.

\begin{tabular}{lccccc}
\hline \multirow{2}{*}{ Item } & \multicolumn{5}{c}{ Factor loading } \\
& & F3 & F1 & F2 & Communality \\
\hline & P6 & $\mathbf{0 . 8 6 8}$ & 0.082 & 0.006 & 0.629 \\
& P5 & $\mathbf{0 . 8 0 0}$ & 0.246 & 0.332 & 0.814 \\
& P1 & $\mathbf{0 . 7 3 6}$ & 0.170 & 0.290 & 0.866 \\
& P3 & $\mathbf{0 . 7 0 4}$ & 0.153 & 0.295 & 0.810 \\
& P2 & $\mathbf{0 . 6 3 0}$ & 0.390 & 0.198 & 0.818 \\
& P4 & $\mathbf{0 . 4 6 2}$ & 0.268 & 0.193 & 0.591 \\
& E3 & 0.101 & $\mathbf{0 . 9 1 2}$ & 0.155 & 0.439 \\
& E2 & 0.216 & $\mathbf{0 . 8 7 3}$ & -0.070 & 0.655 \\
& E1 & 0.312 & $\mathbf{0 . 7 1 4}$ & 0.149 & 0.588 \\
& I2 & 0.079 & -0.114 & $\mathbf{0 . 8 9 4}$ & 0.606 \\
Eigenvalues & I1 & 0.343 & 0.161 & $\mathbf{0 . 8 1 7}$ & 0.323 \\
\% of total & I3 & 0.389 & 0.289 & $\mathbf{0 . 5 9 7}$ & 0.811 \\
variance & I4 & 0.352 & 0.352 & $\mathbf{0 . 4 3 8}$ & 0.760 \\
\hline
\end{tabular}

The bold values are $>0.4$.

TABLE 4: Reliability test.

\begin{tabular}{lcc}
\hline & Cronbach's alpha & Items \\
\hline F1 external support & 0.842 & 3 \\
F2 internal support & 0.776 & 4 \\
F3 performance & 0.859 & 6 \\
All & 0.892 & 13 \\
\hline
\end{tabular}

\section{Result}

The questionnaires are evaluated by SPSS. Multiple regressions are used to test our hypotheses. We use the multiple regressions to test the variables of external and internal supports on the performance of implementing ECS (SuccMail).

5.1. Correlation Tests. Before testing the hypothesis by multiple regressions, we ran the correlations between the external support (3 questions), internal support (4 questions), and the performance of implementing SuccMail (7 questions). Table 5 shows the results. Correlations between 35 of the 42 bivariate correlations are significant and ranged from $0.277(p<0.05)$ to $0.604(p<0.01)$.

5.2. Multiple Regressions. For a more comprehensive test, the standard multiple regression (enter method) analysis is conducted to provide different outcomes to test the hypotheses. Table 6 shows that the results of D1 and D2 
TABLE 5: The correlation matrices.

\begin{tabular}{|c|c|c|c|c|c|c|c|}
\hline & E1 & E2 & E3 & I1 & $\mathrm{I} 2$ & I3 & I4 \\
\hline $\mathrm{P} 1$ & $0.345^{* *}$ & $0.295^{*}$ & $0.277^{*}$ & $0.531^{* *}$ & 0.221 & $0.406^{* *}$ & $0.604^{* *}$ \\
\hline $\mathrm{P} 2$ & $0.478^{* *}$ & $0.414^{* *}$ & $0.449^{* *}$ & $0.430^{* *}$ & $0.271^{*}$ & $0.392^{* *}$ & $0.393^{* *}$ \\
\hline P3 & $0.345^{* *}$ & $0.332^{* *}$ & $0.255^{*}$ & $0.423^{* *}$ & $0.270^{*}$ & $0.594^{* *}$ & $0.483^{* *}$ \\
\hline $\mathrm{P} 4$ & $0.356^{* *}$ & 0.194 & $0.355^{* *}$ & $0.469^{* *}$ & 0.115 & $0.344^{* *}$ & 0.190 \\
\hline P5 & $0.504^{* *}$ & $0.378^{* *}$ & $0.349^{* *}$ & $0.590^{* *}$ & $0.352^{* *}$ & $0.542^{* *}$ & $0.458^{* *}$ \\
\hline P6 & $0.329^{* *}$ & $0.286^{*}$ & 0.213 & $0.318^{*}$ & 0.168 & $0.408^{* *}$ & 0.242 \\
\hline
\end{tabular}

${ }^{*} p<0.05$, two-tailed. ${ }^{* *} p<0.01$, two-tailed.

TABLE 6: Regression analysis summary for dimensions.

\begin{tabular}{lccc}
\hline $\begin{array}{l}\text { Independent } \\
\text { variable }\end{array}$ & $\begin{array}{c}\text { Unstandardized coefficients } \\
\mathrm{B}\end{array}$ & $\begin{array}{c}\text { Unstandardized coefficient std. } \\
\text { error }\end{array}$ & $\begin{array}{c}\text { Standardized coefficients } \\
\text { (beta) }\end{array}$ \\
\hline Constant & 1.079 & 0.347 & 0.310 \\
D1 & 0.257 & 0.083 & $0.529 \quad 5.122^{* *}$ \\
D2 & 0.474 & 0.089 & $5.114^{* *}$ \\
Model fit & $R=0.707$ & $R^{2}=0.500$ & $R^{2}$ adjusted $=0.483 \quad F=20.546^{* * *}$ \\
\hline
\end{tabular}

Note. Dependent variable $=\mathrm{D} 3 .{ }^{* *} p<0.01$, two-tailed. ${ }^{* * *} p<0.001$, two-tailed.

TABLE 7: Regression analysis summary for P1.

\begin{tabular}{|c|c|c|c|c|}
\hline $\begin{array}{l}\text { Independent } \\
\text { variable }\end{array}$ & Unstandardized coefficients B & Unstandardized coefficient std. error & $\begin{array}{l}\text { Standardized coefficients } \\
\text { (beta) }\end{array}$ & $t$ value \\
\hline Constant & 1.132 & 0.518 & & $2.186^{*}$ \\
\hline E1 & 0.046 & 0.125 & 0.048 & 0.364 \\
\hline $\mathrm{E} 2$ & 0.130 & 0.159 & 0.141 & 0.82 \\
\hline E3 & -0.159 & 0.152 & -0.181 & -1.046 \\
\hline I1 & 0.343 & 0.138 & 0.385 & $2.484^{* *}$ \\
\hline $\mathrm{I} 2$ & -0.178 & 0.124 & -0.199 & -1.433 \\
\hline $\mathrm{I} 3$ & 0.116 & 0.105 & 0.150 & 1.105 \\
\hline $\mathrm{I} 4$ & 0.425 & 0.121 & 0.425 & $3.523^{* *}$ \\
\hline Model fit & $R=0.697$ & $R^{2}=0.486$ & $R^{2}$ adjusted $=0.419$ & $F=7.289^{* * *}$ \\
\hline
\end{tabular}

Note. Dependent variable $=$ P1. ${ }^{*} p<0.05$, two-tailed. ${ }^{* *} p<0.01$, two-tailed. ${ }^{* * *} p<0.001$, two-tailed.

dimensions are significantly and positively affecting the dimension of performance.

Table 7 shows that the results of I1 and I4 are significantly and positively affecting $\mathrm{P} 1$; the results provide support for Hypothesis 4 and Hypothesis 7. As the whole details will be recorded in the system by SuccMail, there is barely internal conflict inside the enterprise. Hence, using SuccMail to manage and communicate inside of the enterprise can reduce friction and make tasks easier and reasonable and match our expectations.

Table 8 shows that no factor significantly and positively affects P2, which surprised us. It is because, in the correlation analysis, we found that every item is significantly related to communication improvement. In our interviews, we realized that employees who rely on SuccMail are sure SuccMail can help to improve communication. With further discussions, we found that despite the SME support using SuccMail (ECS), it is the employer or the top managers of the SMEs who are the main barrier of implementing SuccMail. In other words, they seldom or even barely use SuccMail. Whether this is the reason why none of the factors significantly and positively affect P2 or because SuccMail itself is a communication platform deserves a further study.
Table 9 shows that the results of I3 and I4 are significantly and positively affect $\mathrm{P} 3$. These results provide support for Hypothesis 6 and Hypothesis 7. After six months, we ended the survey, one of the enterprises reduced their human resource from 34 to 18 , saving nearly $50 \%$ of human resources. Implementing SuccMail in SMEs and hiring the consultant at the same time would be more able to help the employees to adapt using SuccMail. Even hiring the consultant has an extra expense, and putting the cost of human resource reduction into consideration, it is a worthwhile investment. Meanwhile, after implementing SuccMail, the frictions inside the enterprise were reduced; we found that there were redundant employees inside the enterprise. This ultimately allows companies to reduce personnel costs.

Table 10 shows that $\mathrm{I} 1$ is significantly and positively affecting P4. This result provides support for Hypothesis 4. As we mentioned, once SMEs are fully dependent on SuccMail for enterprise management and communication, the whole details of the event are recorded in the system. All the related employees could have timely access to information and update progress which allows reducing the possibility of having an error. As a result, customer complaints will be reduced. On the other hand, the result 
TABLE 8: Regression analysis summary for P2.

\begin{tabular}{|c|c|c|c|c|}
\hline Independent variable & Unstandardized coefficients B & Unstandardized coefficient std. error & Standardized coefficients (beta) & $t$ value \\
\hline Constant & 0.608 & 0.586 & & 1.038 \\
\hline E1 & 0.229 & 0.142 & 0.234 & 1.616 \\
\hline E2 & 0.184 & 0.179 & 0.195 & 1.027 \\
\hline E3 & 0.046 & 0.172 & 0.051 & 0.265 \\
\hline I1 & 0.166 & 0.156 & 0.183 & 1.062 \\
\hline $\mathrm{I} 2$ & 0.103 & 0.14 & 0.113 & 0.736 \\
\hline $\mathrm{I} 3$ & 0.017 & 0.119 & 0.021 & 0.142 \\
\hline $\mathrm{I} 4$ & 0.091 & 0.137 & 0.089 & 0.667 \\
\hline Model fit & $R=0.607$ & $R^{2}=0.368$ & $R^{2}$ adjusted $=0.286$ & $F=4.494^{* *}$ \\
\hline
\end{tabular}

Note. Dependent variable $=$ P2. ${ }^{* *} p<0.01$, two-tailed.

TABle 9: Regression analysis summary for P3.

\begin{tabular}{|c|c|c|c|c|}
\hline Independent variable & Unstandardized coefficients B & Unstandardized coefficient std. error & Standardized coefficients (beta) & $t$ value \\
\hline Constant & 0.978 & 0.555 & & 1.762 \\
\hline E1 & 0.012 & 0.134 & 0.012 & 0.088 \\
\hline $\mathrm{E} 2$ & 0.219 & 0.17 & 0.223 & 1.291 \\
\hline E3 & -0.225 & 0.163 & -0.240 & -1.377 \\
\hline I1 & 0.041 & 0.148 & 0.043 & 0.279 \\
\hline $\mathrm{I} 2$ & -0.056 & 0.133 & -0.059 & -0.424 \\
\hline I3 & 0.419 & 0.113 & 0.506 & $3.715^{* * *}$ \\
\hline $\mathrm{I} 4$ & 0.357 & 0.129 & 0.334 & $2.761^{* *}$ \\
\hline Model fit & $R=0.649$ & $R^{2}=0.482$ & $R^{2}$ adjusted $=0.414$ & $F=7.164^{* * *}$ \\
\hline
\end{tabular}

Note. Dependent variable $=$ P $3 .{ }^{* *} p<0.01$, two-tailed. ${ }^{* * *} p<0.001$, two-tailed.

TABle 10: Regression analysis summary for P4.

\begin{tabular}{|c|c|c|c|c|}
\hline Independent variable & Unstandardized coefficients B & Unstandardized coefficient std. error & Standardized coefficients (beta) & $t$ value \\
\hline Constant & 2.277 & 0.657 & & 3.466 \\
\hline E1 & 0.172 & 0.159 & 0.158 & 1.079 \\
\hline $\mathrm{E} 2$ & -0.230 & 0.201 & -0.220 & -1.142 \\
\hline E3 & 0.275 & 0.193 & 0.276 & 1.42 \\
\hline I1 & 0.573 & 0.175 & 0.567 & $3.269^{* *}$ \\
\hline $\mathrm{I} 2$ & -0.330 & 0.157 & -0.326 & $-2.102^{* *}$ \\
\hline $\mathrm{I} 3$ & 0.098 & 0.134 & 0.111 & 0.732 \\
\hline $\mathrm{I} 4$ & -0.132 & 0.153 & -0.116 & -0.861 \\
\hline Model fit & $R=0.597$ & $R^{2}=0.356$ & $R^{2}$ adjusted $=0.272$ & $F=4.262^{* *}$ \\
\hline
\end{tabular}

Note. Dependent variable $=$ P4. ${ }^{* *} p<0.01$, two-tailed.

shows that I2 significantly and negatively affects $\mathrm{P} 4$, and this confuses us. The correlation test shows that I2 and P4 have no significant relation. Therefore, we rule out the possibility of the suppressor variable [33]. As the currently existing data is not sufficient to discuss this phenomenon, we will focus our further study on this phenomenon in the future.

Table 11 shows that E1 and I1 are significantly and positively affecting $\mathrm{P} 5$; the results provide support for Hypothesis 1 and Hypothesis 4. When SME fully depends on SuccMail as an internal collaboration and management system, the SuccMail user guide will make it easier for users to adapt it. This leads to enhancing the efficiency of performing tasks, which matches our expectations.

Table 12 shows that there is no variable significantly and positively affecting P6. As the earlier correlation tests, we found that E1 and E3 are very significantly and positively related to P6; also E2 and I1 are significantly and positively related. However, based on the fact that this research does not have the case of a manufacturing company, there is no so-called producing product. It is our next focus to contact the manufacturing companies using SuccMail to study these phenomena in the future.

After all the multiple regressions were tested, we found that E2, E3, and I2 do not significantly or positively affect any performance of implementing ECS. Therefore, the results do not provide support for Hypothesis 2, Hypothesis 3, and Hypothesis 5. Through interviews, we find that SuccMail provides online consultation, but it is not instant consultation. Although an external consultant could give advice, this is also not a real-time service. We considered the reason why Hypothesis 2 and Hypothesis 3 could not be supported; this is because they are not thought of as real-time services. As for Hypothesis 5, when we consider all the variables, the 
TABLE 11: Regression analysis summary for P5.

\begin{tabular}{|c|c|c|c|c|}
\hline $\begin{array}{l}\text { Independent } \\
\text { variable }\end{array}$ & Unstandardized coefficients B & Unstandardized coefficient std. error & $\begin{array}{l}\text { Standardized coefficients } \\
\text { (beta) }\end{array}$ & $t$ value \\
\hline Constant & 0.673 & 0.488 & & 1.378 \\
\hline E1 & 0.257 & 0.118 & 0.272 & $2.174^{*}$ \\
\hline $\mathrm{E} 2$ & 0.276 & 0.150 & 0.304 & 1.843 \\
\hline E3 & -0.233 & 0.144 & -0.270 & -1.626 \\
\hline I1 & 0.345 & 0.130 & 0.395 & $2.653^{* *}$ \\
\hline $\mathrm{I} 2$ & 0.019 & 0.117 & 0.022 & 0.163 \\
\hline $\mathrm{I} 3$ & 0.116 & 0.099 & 0.151 & 1.164 \\
\hline $\mathrm{I} 4$ & 0.101 & 0.114 & 0.103 & 0.889 \\
\hline Model fit & $R=0.726$ & $R^{2}=0.527$ & $R^{2}$ adjusted $=0.466$ & $F=8.597^{* * *}$ \\
\hline
\end{tabular}

Note. Dependent variable $=$ P5. ${ }^{*} p<0.05$, two-tailed. ${ }^{* *} p<0.01$, two-tailed. ${ }^{* * *} p<0.001$, two-tailed.

TABLE 12: Regression analysis summary for P6.

\begin{tabular}{|c|c|c|c|c|}
\hline Independent variable & Unstandardized coefficients B & Unstandardized coefficient std. error & Standardized coefficients (beta) & $t$ value \\
\hline Constant & 1.700 & 0.605 & & 2.809 \\
\hline E1 & 0.147 & 0.147 & 0.159 & 1.001 \\
\hline $\mathrm{E} 2$ & 0.248 & 0.185 & 0.280 & 1.335 \\
\hline E3 & -0.214 & 0.178 & -0.254 & -1.203 \\
\hline I1 & 0.154 & 0.161 & 0.180 & 0.955 \\
\hline $\mathrm{I} 2$ & -0.037 & 0.145 & -0.043 & -0.253 \\
\hline $\mathrm{I} 3$ & 0.184 & 0.123 & 0.247 & 1.491 \\
\hline $\mathrm{I} 4$ & 0.027 & 0.141 & 0.028 & 0.188 \\
\hline Model fit & $R=0.486$ & $R^{2}=0.236$ & $R^{2}$ adjusted $=0.137$ & $F=2.83^{*}$ \\
\hline
\end{tabular}

Note. Dependent variable $=$ P6. ${ }^{*} p<0.05$, two-tailed.

degree of support that the companies offer will not affect the performance. We infer that it might be because while SMEs fully rely on SuccMail, SMEs have often provided a certain degree of support already.

\section{Discussion}

An SME competitiveness can come from using ECS. For smoothly implementing ECS and improving the competitiveness of SME, employees should have a certain level of support in the process of implementing ECS. We explored whether the supports or resources available to SME have an impact on their performance in using SuccMail. After verification and analysis, we conclude the following results:

(1) All supports are positive and influential for the implementation of ECS to improve management efficiency. This meets our initial expectations, as there are many challenges in the process of implementing ECS, and previous researches show that the supply of supports can help solve the challenges of implementing ECS.

(2) We distinguish the supports into external supports (from ECS, "SuccMail") and internal supports (from inside of SME), both of which proved to have an impact on whether enterprises can successfully implement ECS and improve management efficiency, which also meets our expectations. Although supports from ECS software are completely different from the supports from inside the enterprise, we believe that both are equally important. However, after actual research, we found that the impact of internal supports from enterprises is far more significant than the impact of the supports from ECS software.

Through interviews, we find that supports provided within the enterprise are more important for employees to use ECS than supports supplied by ECS. By further analysis, we find that, for users of SuccMail, the immediateness and convenience provided by the internal supports of the enterprise are the most important reasons. The next step is to analyse each support and every benefit when all support variables are considered:

(1) With SuccMail user manual, it is not surprising that the work efficiency can be improved.

(2) When companies fully rely on SuccMail for management and communication, it can improve work efficiency, reduce task difficulty, and reduce customer complaints. This is very much in line with our expectations.

(3) Due to instant communication and clear information, when an enterprise fully relies on using SuccMail for management and communication, it can more easily perform tasks, reduce human resources, and reduce internal friction.

(4) Hiring an exclusive SuccMail consultant inside the enterprise can ultimately reduce human resources. According to our experience, when the company 
fully relies on SuccMail for management and communication and hires a dedicated SuccMail consultant, it can ultimately help SMEs reduce human resources by $30-40 \%$, which we will discuss in detail in the next paper.

(5) The online consultation and the external SuccMail expert consultant do not have a significantly positive effect on the performance of SuccMail. The most important reason is that both online consultation and external SuccMail expert consultant are not realtime services. In the workplace, most of the circumstances require timeliness services, and the nonreal-time services are often not effective. Hence, we suggest that the ECS service company should consider real-time services for its software.

(6) As for the enterprise where offering sufficient resources of using the SuccMail has a significantly negative effect on the performance of implementing SuccMail, in Chapter 5, we mentioned that the currently existing data is not sufficient to discuss it. We will focus on this phenomenon in the following study.

(7) Since our research objects do not have a manufacturing company, there is no so-called producing product. We are not surprised that none of the variables are affecting production efficiency. The performance of implementing SuccMail into a manufacturing company is also a research subject for our next study.

(8) In Chapter 5, we find that the supply of any support has a certain degree of influence on the use of SuccMail for improving communication. When putting all the variables together, no variable has a positive and significant impact. Whether this has a certain relationship with the lack of active participation of SME managers in the use of SuccMail is an interesting research topic.

Due to the lack of academic researches about ECS, we spent two years with three SMEs to do the in-depth exploration. We expect that there will be more academic researches about the relation with ECS and SMEs in the future. Nowadays, most of the certain size SMEs are implementing ERP. The Workflow system is developed by Data Systems Consulting Co., Ltd., which is a widely used ERP system in Taiwan. We had linked the Workflow system with SuccMail and published a conference paper [18]. With this linking, the user can input the information with a drop-down list without logging into the Workflow system, and the SuccMail will automatically produce the purchase order, bill of sale, etc., and also the system will automatically update the stock in the Workflow system. After this linking, the number of users of SuccMail has massively increased. Currently, we have recalled over 400 questionnaires and we will publish the research in the future. Second, we are just about to submit another paper of using Latent Dirichlet Allocation with the visual attention to identify e-mail topic. Also, we will collect the feedback and test its performance from the users.
Although we mentioned that the ERP and ECS have fundamental differences, there are some similarities, especially in the progress of implementation and after implementation. Like the ERP, ECS often faces the customization requests from the users. Therefore, the ECS needs to consider customization requests. An overladen ECS will just cause the failure of implementation. Also, whether it is ERP or ECS, its usage is unique to each enterprise. This reasonably explains that all the knowledge to be transferred in the context of successful ECS implementation will not be explicit nor externalized. Each enterprise has its own uniqueness. In order to adapt to the management characteristics of each enterprise, SuccMail emphasizes that each enterprise should have the maximum degree of freedom under the basic framework of SuccMail. Furthermore, during the implementation of ECS, one of the key factors affecting the success of the implementation is the user's absorptive capacity. However, nowadays, the managers of SMEs in Taiwan are too senile to be familiarised with the personal computer. To solve the user's absorptive capacity, the familiarity of the personal computer is required. This means that those managers have to get familiar with the ECS to a certain degree.

\section{Conclusion}

In this study, we proposed a survey questionnaire to evaluate how the internal and external supports of SuccMail can affect the performance of SME. Each support has a positive influence on the implementation of SuccMail and can affect the business performance of SME. Based on our result, the internal supports are more significant than the external supports to impact the business performance of SME. Industry 4.0 is seen as the beginning phase where computers and automation become connected and as an opportunity to increase the efficiency and effectiveness in the manufacturing industry. There are many challenges related to the integration of Industry 4.0; to catch up with Industry 4.0 , it has become inevitable for the SME to implement e-management. The findings of this study will help the SME better carry out e-management by the implementation of ECS with the internal supports.

\section{Appendix}

\section{A. Questionnaire Items}

(i) The questions below are about the supports that make you get easier on using SuccMail (1: significantly disagree, 5: significantly agree).

(1) The external supports from the SuccMail company.

(a) The SuccMail manual is helpful to use. (E1)

(b) Online consultation is helpful to solve your problems of using SuccMail. (E2)

(c) An external SuccMail expert consultant helps use SuccMail. (E3) 
(2) The internal supports of your company.

(a) Your company fully depends on using SuccMail to manage and communicate. (I1)

(b) Your company offers sufficient resources for using SuccMail. (I2)

(c) Your company had hired a full-time consultant for implementing SuccMail. (I3)

(d) The internal conflicts of the enterprise have been reduced by using SuccMail. (I4)

(ii) The questions below are about the effect of SuccMail on your task performance (1: significantly disagree, 5: significantly agree).

(1) Using SuccMail is easier to perform tasks. (P1)

(2) SuccMail is helpful to improve communication. (P2)

(3) SuccMail is helpful to reduce the cost of human resources. (P3)

(4) SuccMail is helpful to reduce customer complaints. (P4)

(5) Using SuccMail can enhance working efficiency. (P5)

(6) Using SuccMail can increase production efficiency. (P6)

\section{Data Availability}

The authors have included data availability statements within the article.

\section{Conflicts of Interest}

The authors declare that there are no conflicts of interest regarding the publication of this paper.

\section{Acknowledgments}

This work was supported by the Japan Advanced Institute of Science and Technology, Professor Yukari Nagai, and the founder of SuccMail Dr. Tzu-Hang Chiang.

\section{References}

[1] M. Ghobakhloo, M. S. Sabouri, T. S. Hong, and N. Zulkifli, "Information technology adoption in small and mediumsized enterprises; an appraisal of two decades literature," vol. 1, no. 7, p. 29, 2011.

[2] B. S. Ragu-Nathan, C. H. Apigian, T. S. Ragu-Nathan, and Q. Tu, "A path analytic study of the effect of top management support for information systems performance," Omega, vol. 32, no. 6, pp. 459-471, 2004.

[3] P. Schubert and S. P. Williams, "Social business readiness survey 2014," University of Koblenz and Landau, Koblenz, Germany, 2015.

[4] A. Suh and G.-W. Bock, "The impact of enterprise social media on task performance in dispersed teams," in Proceedings of the 2015 48th Hawaii International Conference on System Sciences, pp. 1909-1918, Kauai, HI, USA, January 2015.

[5] E. E. Grandon and J. M. Pearson, "Electronic commerce adoption: an empirical study of small and medium US businesses," Information \& Management, vol. 42, no. 1, pp. 197-216, 2004.

[6] P. Bharati and A. Chaudhury, "SMEs and competitiveness: the role of information systems," International Journal of E-Business Research, vol. 5, no. 1, 2009.

[7] C. Leyh, "Critical success factors for ERP projects in small and medium-sized enterprises - the perspective of selected German SMEs," in Proceedings of the 2014 Federated Conference on Computer Science and Information Systems, pp. 1181-1190, Warsaw, Poland, September 2014.

[8] M. Simpson and A. J. Docherty, "E-commerce adoption support and advice for UK SMEs," Journal of Small Business and Enterprise Development, vol. 11, no. 3, pp. 315-328, 2004.

[9] Y. Wu and X. Wang, "A knowledge management solution based on enterprise information portal," in Proceedings of the 2009 International Workshop on Intelligent Systems and Applications, pp. 1-4, Wuhan, China, May 2009.

[10] P. Schubert and J. H. Glitsch, "Adding structure to enterprise collaboration systems: identification of use cases and collaboration scenarios," Procedia Computer Science, vol. 64, pp. 161-169, 2015.

[11] C. A. Ellis, S. J. Gibbs, and R. Gail, "Groupware: some issues and experiences," Communications of the ACM, vol. 34, no. 1, pp. 39-58, 1991.

[12] H. Fuks, A. Raposo, M. A. Gerosa, Pimental, and Mariano, "The 3C collaboration model," in Encyclopedia of E-Collaboration, pp. 637-644, Texas A\&M International University, Laredo, TX, USA, 2008.

[13] J. O'Brien and G. Marakas, Introduction to Information Systems, Wiley, Hoboken, NJ, USA, 15th edition, 2010.

[14] A. McAfee, "Enterprise 2.0: the dawn of emergent collaboration," IEEE Engineering Management Review, vol. 34, no. 3, p. 38, 2006.

[15] T. H. Davenport, "Putting the enterprise into the enterprise system," Harvard Business Review, vol. 76, no. 4, 1998.

[16] H.-W. Chou, Y.-H. Lin, H.-S. Lu, H.-H. Chang, and S.-B. Chou, "Knowledge sharing and ERP system usage in post-implementation stage," Computers in Human Behavior, vol. 33, pp. 16-22, 2014.

[17] S. Rouhani and A. Zare Ravasan, "ERP success prediction: an artificial neural network approach," Scientia Iranica, vol. 20, no. 3, pp. 992-1001, 2013.

[18] Y. Lin, Y. Nagai, T. Chiang, and H. Chiang, "Design and develop artifact for integrating with ERP and ECS based on design science," in Proceedings of the 2020 the 3rd International Conference on Information Science and System, pp. 218-223, Cambridge, UK, March 2020.

[19] R. Soucek and K. Moser, "Coping with information overload in email communication: evaluation of a training intervention," Computers in Human Behavior, vol. 26, no. 6, pp. 1458-1466, 2010.

[20] M. Chui, The Social Economy: Unlocking Value and Productivity through Social Technologies, McKinsey, New York City, NY, USA, 2012, https://www.mckinsey.com/industries/ technology-media-and-telecommunications/our-insights/ the-social-economy.

[21] G. Nairn, "The trouble with office email," 2011.

[22] C. Greeven and S. P. Williams, "Enterprise collaboration systems: addressing adoption challenges and the shaping of sociotechnical systems," IJISPM-International Journal of Information Systems and Project Management, vol. 5, no. 1, pp. 5-23, 2017. 
[23] T. S. Raghunathan and W. R. King, "The impact of information systems planning on the organization," Omega, vol. 16, no. 2, pp. 85-93, 1988.

[24] J.-H. Park, H.-J. Suh, and H.-D. Yang, "Perceived absorptive capacity of individual users in performance of enterprise resource planning (ERP) usage: the case for Korean firms," Information \& Management, vol. 44, no. 3, pp. 300-312, 2007.

[25] M. Steinhüser, S. Smolnik, and U. Hoppe, "Towards a measurement model of corporate social software success-evidences from an exploratory multiple case study," in Proceedings of the 2011 44th Hawaii International Conference on System Sciences, pp. 1-10, Kauai, HI, USA, January 2011.

[26] C. Herzog and A. Richter, "Use cases as a means to support the appropriation of enterprise social software," in Proceedings of the 2016 49th Hawaii International Conference on System Sciences (HICSS), pp. 4072-4081, Koloa, HI, USA, January 2016.

[27] A. Richter and K. Riemer, "Malleable end-user software," Business \& Information Systems Engineering, vol. 5, no. 3, pp. 195-197, 2013.

[28] M. B. Miles and A. M. Huberman, Qualitative Data Analysis: An Expanded Sourcebook, SAGE, Thousand Oaks, CA, USA, 1994.

[29] R. K. Yin, Case Study Research: Design and Methods, SAGE, Thousand Oaks, CA, USA, 2003.

[30] H. F. Kaiser, "An index of factorial simplicity," Psychometrika, vol. 39, no. 1, pp. 31-36, 1974.

[31] J. Spicer and M. B. J. Spicer, Making Sense of Multivariate Data Analysis: An Intuitive Approach, SAGE, Thousand Oaks, CA, USA, 2005.

[32] J. C. Nunnally, Psychometric Theory, McGraw-Hill, New York, NY, USA, 1978.

[33] B. G. Tabachnick and L. S. Fidell, Using Multivariate Statistics, Pearson Education, London, UK, 2013. 